Virginia Commonwealth University VCU Scholars Compass

2013

\title{
Communication: In search of four-atom chiral metal clusters
}

Xinxing Zhang

Johns Hopkins University

Bradley Visser

Technische Universitat Munchen

Martin Tschurl

Technische Universitat Munchen

See next page for additional authors

Follow this and additional works at: http://scholarscompass.vcu.edu/phys_pubs

Part of the Physics Commons

Zhang, X., Visser, B., \& Tschurl, M., et al. Communication: In search of four-atom chiral metal clusters. The Journal of Chemical Physics, 139, 111101 (2013). Copyright (C) 2013 AIP Publishing LLC.

\section{Downloaded from}

http://scholarscompass.vcu.edu/phys_pubs/110

This Article is brought to you for free and open access by the Dept. of Physics at VCU Scholars Compass. It has been accepted for inclusion in Physics Publications by an authorized administrator of VCU Scholars Compass. For more information, please contact libcompass@vcu.edu. 
Authors

Xinxing Zhang, Bradley Visser, Martin Tschurl, Evan Collins, Yi Wang, Qian Wang, Yawei Li, Qiang Sun, Puru Jena, Gerd Gantefoer, Ulrich Boesl, Ulrich Heiz, and Kit H. Bowen Jr. 


\title{
Communication: In search of four-atom chiral metal clusters
}

\author{
Xinxing Zhang, ${ }^{1}$ Bradley Visser, ${ }^{2}$ Martin Tschurl, ${ }^{2}$ Evan Collins, ${ }^{1}$ Yi Wang, ${ }^{1}$ Qian Wang, ${ }^{3,5}$ \\ Yawei Li, ${ }^{4}$ Qiang Sun, ${ }^{4,5}$ Puru Jena,${ }^{5}$ Gerd Gantefoer, ${ }^{6}$ Ulrich Boesl, ${ }^{2}$ Ulrich Heiz, ${ }^{2, a)}$ \\ and Kit Bowen ${ }^{1, a)}$ \\ ${ }^{1}$ Department of Chemistry, Johns Hopkins University, Baltimore, Maryland 21218, USA \\ ${ }^{2}$ Department Chemie, Technische Universität München, Lichtenbergstrasse 4, 85748 Garching, Germany \\ ${ }^{3}$ Center for Applied Physics and Technology, Peking University, Beijing 100871, China \\ ${ }^{4}$ Department of Materials Science and Engineering, College of Engineering, Peking University, Beijing \\ 100871, China \\ ${ }^{5}$ Department of Physics, Virginia Commonwealth University, Richmond, Virginia 23284, USA \\ ${ }^{6}$ Department Physik Universitaet Konstanz, 78457 Konstanz, Germany
}

(Received 27 June 2013; accepted 5 September 2013; published online 17 September 2013)

\begin{abstract}
A combined study utilizing anion photoelectron spectroscopy and density functional theory was conducted to search for four-atom, chiral, metal, and mostly metal clusters. The clusters considered were $\mathrm{AuCoMnBi}{ }^{-/ 0}, \mathrm{AlAuMnO}^{-/ 0}, \mathrm{AgMnOAl}^{-/ 0}$, and $\mathrm{AuAlPtAg}^{-/ 0}$, where the superscripts, ${ }^{-} / 0$, refer to anionic and neutral cluster species, respectively. Based on the agreement of experimentally and theoretically determined values of both electron affinities and vertical detachment energies, the calculated cluster geometries were validated and examined for chirality. Among both anionic and neutral clusters, five structures were identified as being chiral. () 2013 AIP Publishing LLC. [http://dx.doi.org/10.1063/1.4821766]
\end{abstract}

\section{INTRODUCTION}

The properties of metal clusters have been studied extensively over the past decades in the gas phase ${ }^{1-5}$ as well as on surfaces. ${ }^{6-10}$ The primary driving forces behind these studies were the discoveries of the non-scalable size regime and the unique catalytic properties of small metal clusters. ${ }^{11}$ In particular, the latter has fostered the desire to extend the research even to the field of asymmetric catalysis. So far, two approaches have been tried to use metal clusters for the catalysis of particular enantiomers: the first method, which has proven to be very successful for the asymmetric hydrogenation reaction of ketones, is to use metal colloids in combination with a chiral modifier. ${ }^{12}$ The modifier molecules are added to the reaction solution and induce chirality. The second and more recent approach is to protect metal clusters with chiral ligands and induce chirality directly onto the cluster catalyst. Recently, such an approach has been successfully used in the asymmetric Suzuki-Miyaura, coupling reaction. ${ }^{13}$ Both methods, however, have their limitations. In the first approach, the modifier must bind to both the reactant and the cluster, ${ }^{14}$ and there are only a few molecules that fulfill such criteria. In the second approach, the use of chiral ligands to cap the metal clusters strongly limits the reactivity of the catalysts. The binding of ligands to the clusters blocks active sites, which by definition results in a lower activity of the catalyst. In some cases, chiral arrangements of ligand-protected clusters were found, even when the clusters where protected by achiral ligands, ${ }^{15}$ but these circumstances are based on the orientation of gold-sulfur units bound on the surface of an achiral, gold metal core.

\footnotetext{
a)Electronic addresses: kbowen@jhu.edu and ulrich.heiz@mytum.de
}

Clearly, the most versatile approach for asymmetric catalysis would be the use of intrinsically chiral, bare metal clusters. However, limited work has been performed on the identification of intrinsically chiral bare metal clusters thus far, primarily due to the difficulty in isolating single enantiomers. A recent combined experimental/theoretical study by Letchken et al. is significant, since it shows that the $\mathrm{Au}_{34}$ anion is potentially chiral. ${ }^{16,17}$ Furthermore, theoretical studies have investigated other clusters, such as $\mathrm{Au}_{28}$ and $\mathrm{Au}_{55}$, and found chiral ground states. ${ }^{18}$ Even more recently, a theoretical study has shown that small boron hydride clusters have chiral structures. ${ }^{19}$ In contrast to these simple structures, the intrinsically chiral clusters previously studied have relatively complex structures.

According to the definition of chirality, the simplest chiral molecule possible is composed of four different atoms arranged in a three-dimensional geometry, i.e., neither planar nor linear. In this work, four-atom metal and mostly metal cluster anions are generated in the gas phase and investigated by negative ion photoelectron spectroscopy (PES). The experimental electron affinity (EA), vertical detachment energy (VDE), and the photoelectron spectra are compared with theoretical values of the same quantities obtained from calculations based on density functional theory (DFT). The accompanying (validated) cluster geometries are then examined in order to ascertain chirality.

\section{METHODS}

\section{Experimental details}

In the present work, anion photoelectron spectroscopy is conducted by crossing a mass-selected beam of negative ions with a fixed-energy photon beam and energy analyzing the 
resulting photodetached electrons. This technique is governed by the energy-conservation relationship, $\mathrm{h} v=\mathrm{EBE}+\mathrm{EKE}$, where $\mathrm{h} v, \mathrm{EBE}$, and EKE are the photon energy, the electron binding (transition) energy, and the electron kinetic energy, respectively. Our photoelectron spectrometer, which has been described elsewhere, ${ }^{20}$ consists of one of several different anion sources, a linear time-of-flight mass spectrometer, a mass gate, a momentum decelerator, a neodymium-doped yttrium aluminum garnet (Nd:YAG) laser for photodetachment, and a magnetic bottle, electron energy analyzer with a resolution of $35 \mathrm{meV}$ at $\mathrm{EKE}=1 \mathrm{eV}$. Photoelectron spectra were calibrated against the well-known photoelectron spectrum of $\mathrm{Cu}^{-} .^{21}$

The cluster anions in this study were generated in a pulsed arc cluster ionization source (PACIS), which has been described in detail elsewhere. ${ }^{22}$ In brief, the sample cathode was vaporized by applying a $\sim 30 \mu$ s duration, $180 \mathrm{~V}$ electrical discharge pulse between the anode and the sample cathode. The sample cathode was prepared in a nitrogen-filled glove box, where $\mathrm{Au}, \mathrm{Co}, \mathrm{Mn}, \mathrm{Bi}$, or $\mathrm{Al}, \mathrm{Au}, \mathrm{Mn}$, or $\mathrm{Ag}, \mathrm{Mn}$, $\mathrm{Al}$, or $\mathrm{Au}, \mathrm{Al}, \mathrm{Pt}, \mathrm{Ag}$ powder mixtures were firmly pressed into a depression atop an aluminum rod. About 10 bar of ultrahigh purity helium gas was then used to propel the resulting plasma mix down a $20 \mathrm{~cm}$ flow tube, where it reacted, formed clusters, and was cooled. Anions generated by this method were then mass-selected prior to photoelectron spectroscopic studies.

\section{Computational details}

Our calculations were based on spin-polarized density functional theory (DFT) as implemented in the DMol3 package. ${ }^{23}$ A double numerical plus double polarization basis set was used for all atoms, and relativistic effects were included with the all-electron scalar relativity option of VPSR ${ }^{24}$ The generalized gradient approximation (GGA) for exchange-correlation potential in the form of Becke-LeeYang-Parr (BLYP) ${ }^{25}$ was used for all the studied clusters with the exception of MnCoAuBi, where the BLYP functional resulted in poor convergence during structural optimization. For that cluster, calculations were performed using the PW91 26 GGA functional. All the structures were relaxed without any symmetric constraints. The criteria of convergence of energy, force, and displacement were set to be $1 \times 10^{-6} \mathrm{Ha}$, $0.002 \mathrm{Ha} / \AA$, and $0.005 \AA$, respectively. The EA is calculated as the energy difference between the ground states of the anion and the neutral. The VDE, on the other hand, is the energy difference between the anion ground state and neutral having the anion geometry. The photoelectron spectra are simulated by using the extended Koopman's theorem. ${ }^{27}$

\section{RESULTS}

The anion photoelectron spectra of $\mathrm{AuCoMnBi}^{-}$, $\mathrm{AlAuMnO}^{-}, \mathrm{AgMnOAl}^{-}$, and $\mathrm{AuAlPtAg}{ }^{-}$cluster anions are shown in Figures 1(a)-1(d), respectively. The oxygen atoms in some of the resulting cluster compositions originate from air oxidation of the metal powders during transfer. All of these photoelectron spectra were measured using $355 \mathrm{~nm}(3.49 \mathrm{eV})$

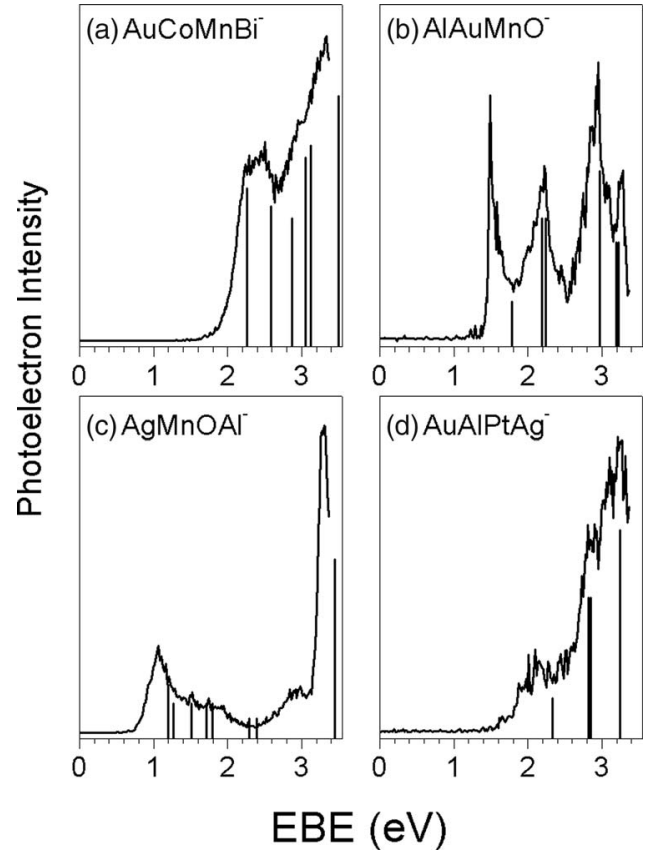

FIG. 1. Photoelectron spectra of (a) $\mathrm{AuCoMnBi}^{-}$, (b) $\mathrm{AlAuMnO}^{-}$, (c) $\mathrm{AgMnOAl}^{-}$, and (d) AuAlPtAg ${ }^{-}$. The theoretical simulated spectra using the extended Koopmans theorem are presented as stick spectra.

photons (third harmonic of the Nd:YAG laser). For each spectrum, both adiabatic electron affinity (EA) and the vertical detachment energy (VDE) values were determined, so they could be compared with our computational results. While the EA value is the energy difference between the lowest energy states of the anion and its neutral counterpart, the VDE value is the photodetachment transition energy at which the Franck Condon overlap between the wave functions of the anion and its neutral counterpart are maximal. Thus, while the EA value can be estimated as the EBE near the intensity threshold, the VDE value corresponds to the EBE at the intensity maximum in a given spectrum. All values of EA and VDE, both measured from the spectra and calculated by theory, are summarized in Table I. The additional higher EBE bands found in each spectrum correspond either to transitions to higher electronic states of the neutral cluster or to photodetachment transitions from different anionic isomers. To simulate the entire photoelectron spectra, we have used the extended Koopman's theorem. ${ }^{27}$ In this approach, the lowest binding energy corresponds to the VDE while

TABLE I. Experimental and theoretical values of electron affinities (EA) and vertical detachment energies (VDE), all presented in units of eV. Experimental values are uncertain by $\pm 0.1 \mathrm{eV}$.

\begin{tabular}{|c|c|c|c|c|}
\hline System & EA (Theo.) & EA (Expt.) & VDE (Theo.) & VDE (Expt.) \\
\hline $\mathrm{AuCoMnBi}^{-}$ & 2.01 & 2.0 & 2.26 & 2.3 \\
\hline $\mathrm{AlAuMnO}^{-}$ & 1.74 & 1.4 & 1.78 & 1.5 \\
\hline $\mathrm{AgMnOAl}^{-}$ & 0.86 & 0.8 & 1.20 & 1.1 \\
\hline $\mathrm{AuAlPtAg}^{-}$iso 1 & 2.15 & 1.9 & 2.33 & 2.1 \\
\hline $\mathrm{AuAlPtAg}^{-}$iso 2 & 1.98 & - & 2.00 & - \\
\hline $\mathrm{AuAlPtAg}{ }^{-}$iso 3 & 1.94 & - & 2.27 & - \\
\hline
\end{tabular}


$\mathrm{AuCoMnBi}$

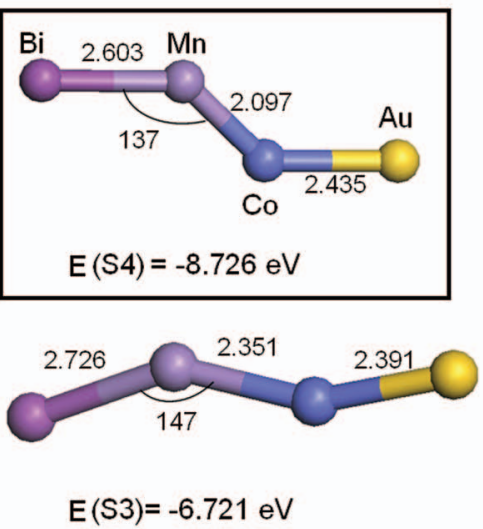

AuAlPtAg Isomer 1

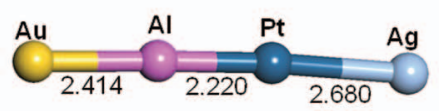

$E(S 1)=-10.518 \mathrm{eV}$

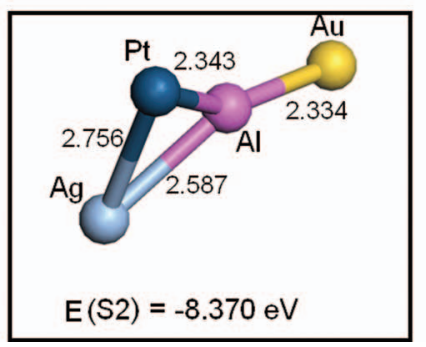

AlAuMnO
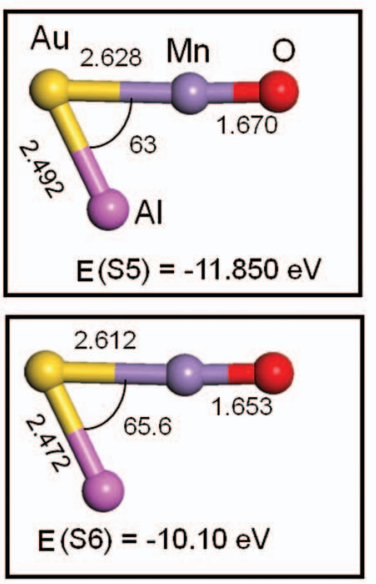

AuAlPtAg Isomer 2

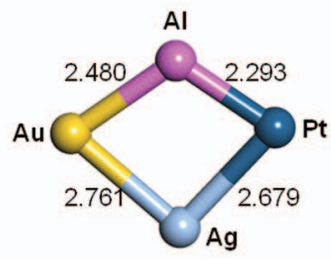

$E(S 1)=-10.517 \mathrm{eV}$

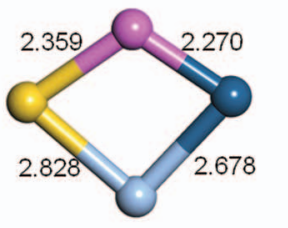

$E(S 2)=-8.538 \mathrm{eV}$
$\mathrm{AgMnOAl}$

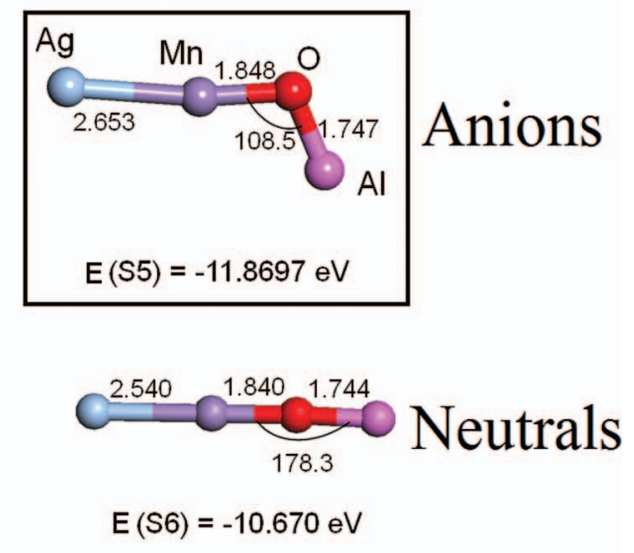

AuAlPtAg Isomer 3
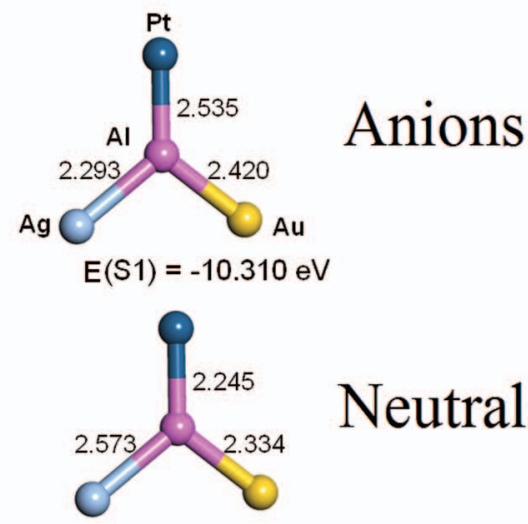

Neutrals

FIG. 2. Calculated structures and relative energies of $\mathrm{AuCoMnBi}^{-/ 0}, \mathrm{AlAuMnO}^{-/ 0}, \mathrm{AgMnOAl}^{-/ 0}$, and the three isomers of $\mathrm{AuAlPtAg}^{-/ 0}$. The chiral structures are indicated by boxes drawn around them.

successive energies are calculated by adding the energy difference between the HOMO and HOMO-i $(i=1,2,3, \ldots)$ levels to the VDE. This is similar to the use of electronic density of states where the molecular orbital energy levels are broadened by a Gaussian. ${ }^{27}$

DFT calculations indicate that $\mathrm{AuAlPtAg}^{-}$has three, nearly degenerate isomers, i.e., isomer 1,2 , and 3 . Their corresponding EA and VDE values are listed separately in Table I. For each of the other three systems, however, only one low-lying isomer is found. The calculated lowest energy structures of all four systems are presented in Figure 2, these including the structures of both anionic and their corresponding neutral clusters.

\section{DISCUSSION}

The good agreement between experimental and computed values of EA and VDE as well as the experimental and simulated PES validates the computed minimum energy, geometric structures. Furthermore, the computed geometry differences between anions and their corresponding neutrals are consistent with their observed photoelectron spectra. For example, in the cases of $\mathrm{AlAuMnO}$ and $\mathrm{AlAuMnO}^{-}$, where both the anion and neutral structures are calculated to be very similar, the lowest EBE peak is very narrow.

For AuAlPtAg ${ }^{-}$, three distinct isomers are found through our calculations. The computed EA values are all in the same range, i.e., $2.15 \mathrm{eV}, 1.98 \mathrm{eV}$, and $1.94 \mathrm{eV}$, making it difficult to distinguish the presence of one from another. Thus, all of these anionic isomers could be contributing to the observed spectra, with their transitions superimposed. In fact, the features in the AuAlPtAg ${ }^{-}$spectrum do indeed show substantial broadening, consistent with this possibility.

The focus of this work is on the search for chiral, metal, or mostly metal clusters. Since it is difficult to tell whether a given cluster is planar or not in two-dimensional figures, we have given the coordinates of all the cluster structures in their Z-matrices. ${ }^{28}$ For all of these clusters, the four atoms are labeled by numbers $(1,2,3$, and 4$)$. The bond angle, $\angle 123$ is written as $\theta_{1}$ and the bond angle, $\angle 234$ as $\theta_{2}$. The dihedral angle between the two planes, defined by atoms 1, 2, 3 and atoms $2,3,4$, respectively, are denoted by $\Phi$. The angles for 
TABLE II. Bond angles $\left(\theta_{1}, \theta_{2}\right)$ and dihedral angles $(\Phi)$ for all of the four systems.

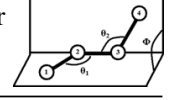

Systems (1, 2, 3,

\begin{tabular}{|c|c|c|c|c|}
\hline 4) & $\theta_{1}$ & $\theta_{2}$ & $\Phi$ & Chirality \\
\hline $\mathrm{AuCoMnBi}$ & 155.16 & 146.92 & 180.0 & No \\
\hline $\mathrm{AuCoMnBi}^{-}$ & 136.44 & 137.08 & -176.92 & Yes \\
\hline AlAuMnO & 65.61 & 177.54 & -95.81 & Yes \\
\hline $\mathrm{AlAuMnO}^{-}$ & 62.90 & 176.95 & -115.70 & Yes \\
\hline $\mathrm{AgMnOAl}$ & 179.84 & 178.28 & -14.21 & No \\
\hline $\mathrm{AgMnOAl}^{-}$ & 172.06 & 108.45 & 176.88 & Yes \\
\hline AuAlPtAg iso 1 & 151.37 & 139.36 & 173.88 & Yes \\
\hline $\mathrm{AuAlPtAg}^{-}$iso 1 & 179.87 & 179.84 & -169.95 & No \\
\hline AuAlPtAg iso 2 & 121.96 & 73.5 & 180.0 & No \\
\hline $\mathrm{AuAlPtAg}^{-}$iso 2 & 110.03 & 81.7 & 180.0 & No \\
\hline AuAlPtAg iso 3 & 153.75 & 136.87 & 180.0 & No \\
\hline $\mathrm{AuAlPtAg}^{-}$iso 3 & 128.69 & 102.45 & 180.0 & No \\
\hline
\end{tabular}

all four clusters are shown in Table II, and their atoms are labeled in sequential order, i.e., 1, 2, 3, 4. For example, in $\mathrm{AuCoMnBi}$ (neutral), $\mathrm{Au}, \mathrm{Co}, \mathrm{Mn}$, and $\mathrm{Bi}$ atoms are respectively labeled as atom number; 1, 2, 3, and 4. Accordingly, $\theta_{1}$ is $\angle \mathrm{AuCoMn}, \theta_{2}$ is $\angle \mathrm{CoMnBi}$, and $\Phi$ is the dihedral angle between the two planes, defined by atoms, AuCoMn and CoMnBi. A cluster is chiral if the geometry of the species is non-planar (and nonlinear), and thus, none of the three angles is $180^{\circ}$. Five of the calculated structures were identified as chiral (see structures enclosed in boxes in Figure 2). These chiral clusters are $\mathrm{AuCoMnBi}^{-}, \mathrm{AlAuMnO}^{-/ 0}, \mathrm{AgMnOAl}^{-}$, and $\mathrm{AuAlPtAg}{ }^{0}$ (isomer 1). Note that in the case of AlAuMnO and its anion, $\mathrm{AlAuMnO}^{-}$, the structures of both are chiral.

Nevertheless, since deviations from planarity are small $\left(<4^{\circ}\right)$ in all the structures studied here, the uncertainty in our ability to calculate angles must be addressed. Typically, we believe the reliability of the angles calculated here is good to within $\sim 0.1^{\circ}$. This standard allowed us to comfortably identify the five clusters listed above as being chiral. However, this same criterion also required us not to include two clusters, i.e., $\mathrm{AgMnOAl}^{0}$ and $\mathrm{AuAlPtAg}^{-}$(isomer 1), which based on the numerical value of their angles alone might have been designated as chiral.

The combination of anion photoelectron spectroscopic experiments and DFT calculations have led to the identification of five metal or mostly metal clusters as being chiral. Thus, with further study and development, the prospects seem bright for intrinsically chiral, bare metal, and/or mostly metal clusters becoming available for research in asymmetric catalysis.

\section{ACKNOWLEDGMENTS}

Parts of this study are based upon work supported by the National Science Foundation under Grant No.
CHE-1111693 (KHB), by an ERC Advanced Grant (ERC2009-AdG 246645-ASC3), and by the U.S. Department of Energy, Office of Basic Energy Sciences, Division of Materials Sciences and Engineering under Award No. DE-FG0296ER45579 (P.J.).

${ }^{1}$ A. Herrmann, E. Schumacher, and L. Wöste, J. Chem. Phys. 68, 2327 (1978).

${ }^{2}$ W. D. Knight, K. Clemenger, W. A. de Heer, W. A. Saunders, M. Y. Chou, and M. L. Cohen, Phys. Rev. Lett. 52, 2141 (1984).

${ }^{3}$ K. M. McHugh, J. G. Eaton, G. H. Lee, H. W. Sarkas, L. K. Kidder, J. T. Snodgrass, M. R. Manaa, and K. H. Bowen, J. Chem. Phys. 91, 3792 (1989).

${ }^{4}$ L. Zheng, C. M. Karner, P. J. Brucat, S. H. Yang, C. L. Pettiette, M. J. Craycraft, and R. E. Smalley, J. Chem. Phys. 85, 1681 (1986).

${ }^{5}$ E. A. Rohlfing, D. M. Cox, and A. Kaldor, Chem. Phys. Lett. 99, 161 (1983).

${ }^{6}$ A. Sanchez, S. Abbet, U. Heiz, W-D. Schneider, H. Häkkinen, R. N. Barnett, and Uzi Landman, J. Phys. Chem. A 103, 9573 (1999).

${ }^{7}$ U. Heiz, A. Sanchez, S. Abbet, and W-D. Schneider, J. Am. Chem. Soc. 121, 3214 (1999).

${ }^{8}$ A. Kartouzian, M. Thämer, T. Soini, J. Peter, P. Pitschi, S. Gilb, and U. Heiz, J. Appl. Phys. 104, 124313 (2008).

${ }^{9}$ K. Bromann, C. Félix, H. Brune, W. Harbich, R. Monot, J. Buttet, and K. Kern, Science 274, 956 (1996).

${ }^{10}$ B. Wang, B. Yoon, M. König, Y. Fukamori, F. Esch, U. Heiz, and U. Landman, Nano Lett. 12, 5907 (2012).

${ }^{11}$ U. Heiz and U. Landman, Nanocatalysis, 1st ed. (Springer, 2006).

${ }^{12}$ H. Bonnemann and G. A. Braun, Angew. Chem., Int. Ed. 35, 1992 (1996).

${ }^{13}$ K. Sawai, R. Tatumi, T. Nakahodo, and H. Fujihara, Angew. Chem., Int. Ed. 47, 6917 (2008).

${ }^{14}$ H. U. Blaser and M. Studer, Acc. Chem. Res. 40, 1348 (2007); T. Mallat, E. Orglmeister, and A. Baiker, Chem. Rev. 107, 4863 (2007); M. Sutyinszki, K. Szori, K. Felfoldi, and M. Bartok, Catal. Commun. 3, 125 (2002); T. Burgi and A. Baiker, Acc. Chem. Res. 37, 909 (2004).

${ }^{15}$ S. Knoppe, I. Dolamic, A. Dass, and T. Bürgi, Angew. Chem., Int. Ed. 51, 7589 (2012); I. Dolamic, S. Knoppe, A. Dass and T. Bürgi, Nat. Commun. 3, 798 (2012).

${ }^{16}$ A. Lechtken, D. Schooss, J. R. Stairs, M. N. Blom, F. Furche, N. Morgner, O. Kostko, B. von Issendorff, and M. M. Kappes, Angew. Chem., Int. Ed. 46, 2944 (2007).

${ }^{17}$ X. Gu, S. Bulusu, X. Li, X. C. Zeng, J. Li, X. G. Gong, and L.-S. Wang, J. Phys. Chem. C 111, 8228 (2007).

${ }^{18}$ I. L. Garzón, J. A. Reyes-Nava, J. I. Rodríguez-Hernández, I. Sigal, M. R. Beltrán, and K. Michaelian, Phys. Rev. B 66, 073403 (2002).

${ }^{19}$ J. K. Olson, and A. I. Boldyrev, Chem. Phys. Lett. 517, 62 (2011).

${ }^{20}$ M. Gerhards, O. C. Thomas, J. M. Nilles, W. J. Zheng, and K. H. Bowen, J. Chem. Phys. 116, 10247 (2002).

${ }^{21}$ J. Ho, K. M. Ervin, and W. C. Lineberger, J. Chem. Phys. 93, 6987 (1990).

${ }^{22}$ X. Li, A. Grubisic, S. T. Stokes, J. Cordes, G. F. Gantefoer, K. H. Bowen, B. Kiran, M. Willis, P. Jena, R. Burgert, and H. Schnöckel, Science 315, 356 (2007)

${ }^{23}$ Molecular Simulations, DMol3, Molecular Simulations Inc., San Diego, CA, 2001.

${ }^{24}$ B. Delley, Int. J. Quantum Chem. 69, 423 (1998).

${ }^{25}$ C. Lee, W. Yang, and R. G. Parr, Phys. Rev. B 37, 785 (1988).

${ }^{26}$ J. P. Perdew, J. A. Chevary, S. H. Vosko, K. A. Jackson, M. R. Pederson, D. J. Singh, and C. Fiolhais, Phys. Rev. B 46, 6671 (1992); 48, 4978 (1993).

${ }^{27}$ A. K. Kandalam, B. Kiran, P. Jena, X. Li, A. Grubisic, and K. H. Bowen, J. Chem. Phys. 126, 084306 (2007); Lei-Ming Wang and Lai-Sheng Wang, Nanoscale 4, 4038 (2012).

${ }^{28}$ M. S. Gordon and J. A. Pople, J. Chem. Phys. 49, 4643 (1968). 